\title{
Adding the merits of contrast to the ease of mammography; can we highlight what's behind breast asymmetries?
}

\author{
Rasha Mohamed Kamal ${ }^{1}$, Amr Farouk Ibrahim Moustafa ${ }^{2 *}$ (D), Sherihan Fakhry ${ }^{1}$, Eman Faker Kamal ', Amira Radwan², \\ Amany Hilal ${ }^{3}$ and Maher Hassan ${ }^{4}$
}

\begin{abstract}
Background: Breast symmetry is one of the major things that radiologists assess when looking at mammograms and is one of the most challenging mammographic findings to evaluate. Contrast-enhanced spectral mammography (CESM) is an emerging mammography technique that has shown comparable sensitivity and specificity to MRI. The purpose of this study is to assess the value of CESM in characterization of breast asymmetries (BAs) and if it should be incorporated in its diagnostic work-up.

Results: Three hundred sixty-five patients with mean age of 47 years were included in the study. CESM was performed aiming for characterization of 380 suspicious or indeterminate breast asymmetries. Assessment of subtracted high-energy images (HEI) markedly improves the overall accuracy reaching $88.4 \%$. Further improvement of the overall accuracy was achieved on combined assessment of the low-energy images (LEI), subtracted high-energy images (HEl), and ultrasound reaching 91.3\%.

Conclusion: CESM is considered as a valuable complementary imaging tool considering the evaluation of breast asymmetries and should be incorporated in its diagnostic work-up in cases not resolved on an initial combined mammography and targeted ultrasound study especially in the presence of a heterogeneous dense breast parenchyma. Yet, this may be hindered in the presence of inflammatory signs because of the overlapping imaging criteria.
\end{abstract}

Keywords: Contrast-enhanced spectral mammogram, Breast asymmetry

\section{Background}

Breast symmetry is one of the major things that radiologists assess when looking at mammograms, and it has been renowned for improved ease and accuracy of mammography interpretation. Radiologists usually perceive both breasts as symmetric in size and distribution of fibroglandular tissue $[1,2]$. Breast asymmetries (BAs) can be quite normal and are often secondary to variations in normal breast tissue. Nevertheless, in some cases, they may indicate an underlying serious pathology $[1,3]$.

Breast asymmetries are one of the most challenging mammographic findings to evaluate, and they often

\footnotetext{
* Correspondence: amrfaroukmoustafa@cu.edu.eg

${ }^{2}$ Department of Diagnostic and Interventional Radiology, National Cancer Institute, Cairo University, 1 Kasr Elainy Street, Fom Elkalig, Cairo, Egypt Full list of author information is available at the end of the article
}

entail further assessment with additional mammography views and a targeted ultrasound study [4-6]. Ultrasound can provide useful information when a correlate is found, but the situation becomes complicated when no correlate is identified and an underlying malignancy could not be dismissed. Further imaging such as dynamic contrast-enhanced MRI might be requested, and sometimes, a short-term follow-up protocol may be adopted in less suspicious asymmetries [7-9].

Contrast-enhanced spectral mammography (CESM) is an emerging mammography technique that has shown comparable sensitivity and specificity to MRI, yet potentially, it is easier, faster, and more cost-effective [10]. The purpose of this study is to assess the impact of incorporating CESM in the characterization and diagnostic work-up of BAs. 


\section{Methods}

The study is a prospective analytical study which included 365 females with 380 BAs. The study was approved by the ethical committee of a multidisciplinary "Breast Cancer Hospital," and an informed written consent was taken from all subjects.

\section{Subjects}

The subjects in this study were females with indeterminate and suspicious BAs that were not completely resolved with mammography and ultrasound. The commonest complaint for younger age group was mastalgia followed by breast lump, while in older age group, it was mostly following screening mammogram. Mammograms performed were both diagnostic and screening exams. CESM was requested aiming both for asymmetry characterization and for identifying the extent of underlying lesions.

\section{Technique of CESM}

A single shot of $1.5 \mathrm{~mL} / \mathrm{kg}$ of non-ionic iodinated contrast agent was injected. Compression was then applied starting by the contralateral side of concern to increase the likelihood of contrast uptake by the breast of concern. A pair of low $(26-30 \mathrm{kVp})$ and high $(45-49 \mathrm{kVp})$ energy exposures during each mammographic position was taken. A weighted subtraction was performed automatically generating an image with enhanced conspicuity of contrast agent uptake.

\section{Image evaluation}

Distinct analysis of the low- and the high-energy images was accomplished by independent double reading by 2 different breast imaging radiologists with at least 10 years' experience in mammography and 5 and 7 years' experience in contrast mammography. Readers were blinded to each other's analysis and to ultrasound results. In case of disagreement between the two radiologists, the mammograms were re-evaluated in consultation and agreement was achieved. Findings were then correlated with histopathology results of core and operative specimens and/or follow-up outcomes for at least 18 months to ascertain a benign diagnosis.

The low-energy mammography images (LEMI) were evaluated in accordance to the 2013 mammography BIRADS lexicon [5], the breast density was recorded, the type of asymmetry was specified (single view, global, focal, or developing), and the associated features were reported (microcalcifications, skin thickening, associated parenchymal distortion, and axillary lymph node status).

The contrast images were evaluated in accordance to the 2013 MRI BI-RADS lexicon as there is no CESMspecified lexicon [11]. The degree of background parenchymal enhancement (BPE) was recorded, and lesions were classified into non-enhancing and enhancing (focus, mass, and non-mass). Breast asymmetries with underlying non-enhancing lesions and enhancing lesions showing benign morphology descriptors were classified as benign and were subjected to a short-term follow-up study for at least 18 months. Lesions that showed an increase in size or conspicuity on follow-up studies were biopsied. Asymmetries with underlying enhancing lesions showing malignant morphology descriptors were subjected to immediate core biopsy.

\section{Statistical analysis}

Data were coded and entered using the statistical package SPSS (Statistical Package for the Social Sciences) version 24. Data was summarized using frequency (count) and relative frequency (percentage) for categorical data. Standard accuracy measures including positive and negative likelihood ratios and overall accuracy were calculated for the different diagnostic tests. The degree of uncertainty of the accuracy measures was described by calculating the 95\% confidence interval (CI). For comparing categorical data, chi-square $\left(\chi^{2}\right)$ test was performed. $p$ value less than 0.05 was considered significant.

\section{Results}

We prospectively analyzed the CESM findings of 380 suspicious or indeterminate BAs in 365 patients who attended a "Multidisciplinary Breast Cancer Hospital." Fifteen patients had multiple lesions: 5 patients presented 2 asymmetries in the same breast and 10 patients presented bilateral asymmetries (Fig. 1). Their ages ranged from 29 to 69 years (mean age 47 years).

According to histopathology results and outcomes of follow-up studies, 35/380 (9.2\%) asymmetries were overlapping tissues, 88/380 (23.2\%) were benign lesions, and 257/380 (67.6\%) were malignant lesions. Biopsy was performed for 286 asymmetries: instantly in 269 cases and postponed to the follow-up study in 17 cases that showed an increase in lesion size and/or conspicuity (Table 1).

The breast density was assessed on the LEMI: 100/365 (27.4\%) cases were classified as ACR "a" or "b" and 265/ 365 (72.6\%) were classified as ACR "c" or "d."

Asymmetries were then classified into single view (20/380, 5.3\%), global (128/380, 33.7\%), focal (228/ $380,60 \%)$, and developing (4/380, 1\%). The presence of a global, focal, or developing asymmetry strongly correlated with an underlying malignant pathology $(p$ value 0.001 ; Table 2). Associated mammography findings (edema, skin thickening, parenchymal distortion, and calcifications) were seen in 106/365 (29\%) cases.

On evaluating the LEMI, a considerable number of false-negative $(44 / 380,11.6 \%)$ and false-positive (66/380, $17.4 \%)$ asymmetries were encountered. The commonest 


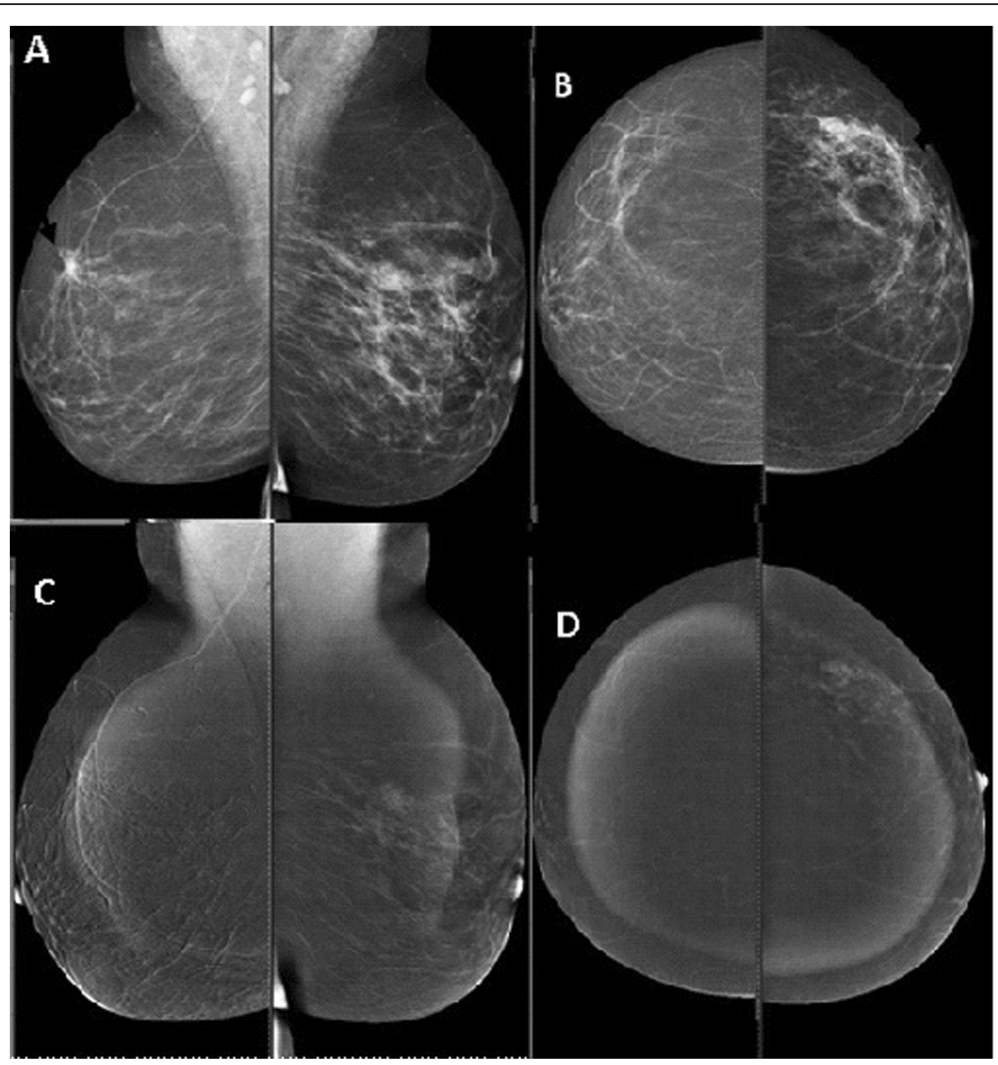

Fig. 1 Right breast focal asymmetry (straight arrow) (a). Left breast global asymmetry (curved arrow) (b). The right breast asymmetry was an adenotic nodule and did not show contrast uptake (c) while the left breast asymmetry was an invasive lobular carcinoma and showed heterogeneous non-mass contrast uptake (d)

Table 1 Classification of the 380 breast asymmetries according to histopathology results and outcome of follow-up studies

\begin{tabular}{lll}
\hline Pathology & Number & Percentage \\
\hline Normal overlapping parenchyma & 35 & 9.2 \\
Benign pathology & 88 & 22.4 \\
- Fibroadenoma & 21 & 23.9 \\
- Inflammatory lesion & 24 & 27.2 \\
- Fibroadenosis & 16 & 18.2 \\
- Fibrocystic changes & 14 & 15.9 \\
- Benign precancerous & 5 & 5.7 \\
- Operative bed & 8 & 9.1 \\
Malignant pathology & $257^{*}$ & $68.4^{*}$ \\
- DCIS & 16 & 6.3 \\
- IDC & 202 & 78.6 \\
- ILC & 25 & 9.7 \\
- Others & 14 & 5.4 \\
\hline
\end{tabular}

*Underlying malignant lesions are significantly higher than normal and benign lesions $(p$ 0.001) cause for the false-negative asymmetries is the heterogeneous dense breast parenchyma $(27 / 44,61.4 \%)$. The commonest causes of false-positive asymmetries are the heterogeneous dense breast $(23 / 66,34.8 \%)$ and the inflammatory breast lesions $(21 / 66,31.8 \%)$ (Table 3$)$. The LEMI showed a positive and negative likelihood ratio of 1.54 (95\% CI 1.30-1.84) and 0.37 (95\% CI 0.27-0.51), respectively, and an overall accuracy of $71 \%$ (95\% CI 66.2-75.5) (Table 4).

On performing a targeted ultrasound examination, no ultrasound correlate was seen in 90/380 (23.7\%) asymmetries, an indeterminate ultrasound correlate was identified in 61/380 (16\%) asymmetries, and a probably malignant ultrasound correlate was identified in 229/380 (60.3\%) (Figs. 2 and 3). The heterogeneous dense parenchyma and inflammatory breast lesions still constitute

Table $\mathbf{2}$ Correlation between the type of asymmetry and its characterization

\begin{tabular}{|c|c|c|c|c|c|c|c|c|}
\hline \multirow[b]{2}{*}{ Normal } & \multicolumn{2}{|c|}{ Simple } & \multicolumn{2}{|c|}{ Global } & \multicolumn{2}{|c|}{ Focal } & \multicolumn{2}{|c|}{ Developing } \\
\hline & 7 & $35 \%$ & 5 & $3.9 \%$ & 23 & $10.0 \%$ & 0 & - \\
\hline Benign & 4 & $20 \%$ & 26 & $20.3 \%$ & 58 & $25.4 \%$ & 0 & - \\
\hline Malignant & 9 & $45 \%$ & 97 & $75.8 \%$ & 147 & $64.6 \%$ & 4 & $100 \%$ \\
\hline Total & 20 & $100 \%$ & 128 & $100 \%$ & 228 & $100 \%$ & 4 & $100 \%$ \\
\hline
\end{tabular}


Table 3 Comparison between the number and frequency of the false-positive and false-negative lesions using the different imaging modalities

\begin{tabular}{|c|c|c|c|c|}
\hline & Mammography & Sonomammogram & CESM & Combined \\
\hline False-negative lesions & 44 lesions & 28 lesions & 14 lesions & 3 lesions \\
\hline - Heterogeneous dense parenchyma & $27 / 44,61.4 \%$ & $20 / 28,71.5 \%$ & - & - \\
\hline - Single view asymmetries & $8 / 44,18.2 \%$ & $1 / 28,3.6 \%$ & - & - \\
\hline - Operative bed recurrence & $4 / 44,9.1 \%$ & $2 / 28,7.1 \%$ & - & - \\
\hline - Non-calcified DCIS & $3 / 44,6.8 \%$ & $3 / 28,10.7 \%$ & - & - \\
\hline - Bilateral asymmetries & $2 / 44,4.5 \%$ & $2 / 28,7.1 \%$ & - & - \\
\hline - Non-enhancing malignant lesions & - & - & $9 / 14,64.3 \%$ & $2 / 3,66.6 \%$ \\
\hline - Faintly enhancing lesions & - & - & $5 / 14,35.7 \%$ & $1 / 3,33.4 \%$ \\
\hline False-positive lesions & 66 lesions & 46 lesions & 30 lesions & 30 lesions \\
\hline - Heterogeneous dense breast & $23 / 66,34.8 \%$ & $15 / 46,32.6 \%$ & - & - \\
\hline - Inflammatory lesions & $21 / 66,31.8 \%$ & $15 / 46,32.6 \%$ & $17 / 30,56.7 \%$ & $17 / 30,56.7 \%$ \\
\hline - Benign masses & $11 / 66,16.7 \%$ & $5 / 46.10 .9 \%$ & - & - \\
\hline - Benign precancerous lesions & $4 / 66,6.1 \%$ & $4 / 46,8.7 \%$ & $3 / 30,10.0 \%$ & $3 / 30,10.0 \%$ \\
\hline - Asymmetric breasts & $4 / 66,6.1 \%$ & $4 / 46,8.7 \%$ & - & - \\
\hline - Operative bed scarring & $3 / 66,4.5 \%$ & $3 / 46,6.5 \%$ & - & - \\
\hline - Benign enhancing lesions & - & - & $10 / 30,33.3 \%$ & $10 / 30,33.3 \%$ \\
\hline
\end{tabular}

the majority of cases. After adding the targeted ultrasound evaluation, the number of false-negative and false-positive cases was reduced to become 28/380 $(7.8 \%)$ and $46 / 380(12 \%)$, respectively. Combining the results of targeted ultrasound and LEMI showed a positive and negative likelihood ratio of 2.38 (95\% CI 1.89-3.01) and 0.17 (95\% CI $0.12-0.25$ ), respectively, and an overall accuracy of $80.53 \%$ (95\% CI 67.18-84.3).

The first step in evaluating the contrast images was to assess the correlation between the breast density and the background parenchymal enhancement (BPE). A very weak positive correlation coefficient was calculated between the breast density and the intensity of the BPE ( $R$ $0.3704, p$ value 0.00001$)$ as $300 / 365(82.2 \%)$ cases showed minimal and mild BPE in comparison to only 65/365 $(17.8 \%)$ cases that showed moderate and severe BPE.

Contrast enhancement was identified in 293/380 asymmetries. The presence of enhancing lesions underlying asymmetries was significantly higher in malignant than in benign lesions ( $p$ value 0.05 ).
After evaluating the contrast images, the number of false-negative and false-positive cases was further reduced to become $14 / 380(3.7 \%)$ and $30 / 380$ (7.9\%), respectively. Non-enhancing malignant tumors $(9 / 14$, $64.3 \%$ ) constituted the majority of false-negative results, and the inflammatory breast lesions (17/30, $56.7 \%)$ and enhancing benign lesions (10/30, 33.3\%) constituted the majority of false-positive cases. The calculated positive and negative likelihood ratio of the contrast images was 3.88 (95\% CI 2.84-5.30) and 0.07 (95\% CI 0.04-0.12), respectively, and an overall accuracy was $88.4 \%$ (95\% CI 84.7-91.4) (Table 4).

The actual extension of malignant asymmetries for staging and pre-operative planning was also evaluated on the contrast images (Fig. 4). Additional lesions within the same breast were encountered in 5/257 malignant asymmetries, and in the contra lateral breast in 10/257 malignant asymmetries. A wider extension was also identified on the contrast images in $42 / 257$ asymmetries (Figs. 3 and 4).

Table 4 The positive predictive value, negative predictive value, and overall accuracy of different imaging modalities in assessment of breast asymmetries

\begin{tabular}{llll}
\hline & Positive predictive value & Negative predictive value & Overall accuracy (\%) \\
\hline Mammography & 1.54 & 0.37 & 71 \\
Sonomammography & 2.38 & 0.17 & 80.53 \\
CESM & 3.88 & 0.07 & 88.4 \\
Combined & 4.05 & 0.02 & 91.32 \\
\hline
\end{tabular}




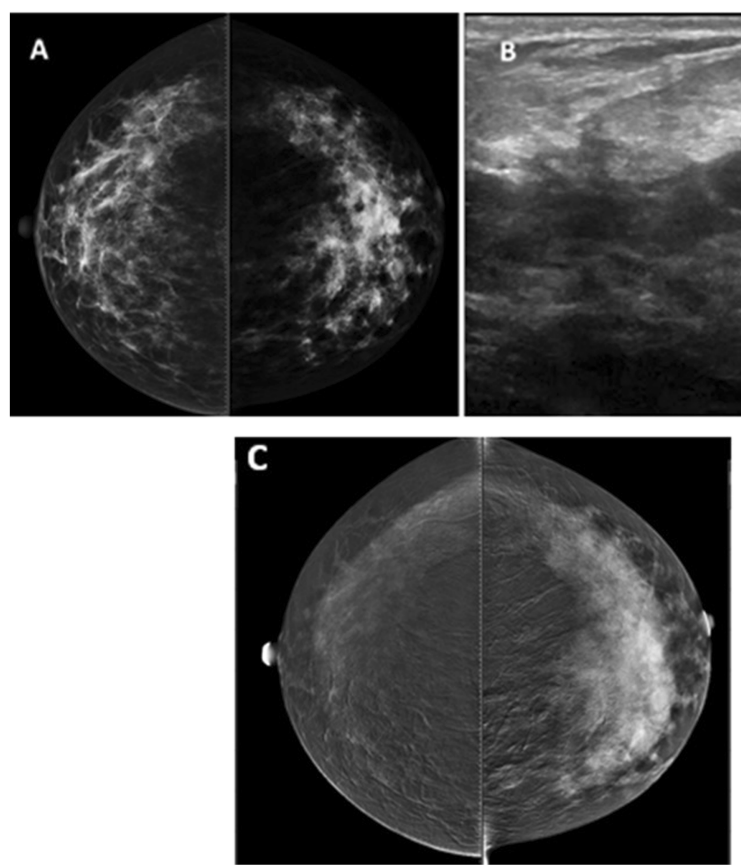

Fig. 2 Global asymmetry of the left breast (a). Ultrasound showed subtle diffusely altered parenchyma (indeterminate ultrasound correlate) (b). CESM showed diffuse heterogeneous malignant non-mass enhancement (c). Biopsy revealed invasive lobular carcinoma

Combining the assessment of the contrast images and complementary ultrasound reduced the falsenegative lesions $(3 / 380,0.78 \%)$ and had no effect on the false-positive lesions $(30 / 380,7.9 \%)$ that were mainly attributed to the inflammatory breast lesions (17 cases) (Fig. 5). The calculated positive and negative likelihood ratio of the combined assessment was 4.05 (95\% CI 2.97-5.53) and 0.02 (95\% CI 0.00-0.05), respectively, and an overall accuracy was $91.32 \%$ (95\% CI 88.02-93.95).

\section{Discussion}

The diagnostic work-up of BAs poses a diagnostic challenge. When asymmetries appear on mammograms, they usually entail further assessment by supplementary imaging tools including extra mammography views, breast ultrasound, and sometimes even MRI [3].

The advanced mammography techniques have offered less expensive and less time-consuming alternatives to MRI. One of these techniques is the CESM which has comparable sensitivity and specificity to MRI. CESM combines the ease and the ability of digital mammography to characterize microcalcifications together with the merits of contrast injection and high sensitivity of MRI [12]. Previous studies have proved that CESM is superior to mammography in the identification of multiplicity, extent, and size of malignant lesions especially in the dense breast parenchyma [10, 13-15].
In the current study, we aimed to assess the impact of incorporating CESM in the characterization and diagnostic work-up of BAs in 365 females with 380 indeterminate and suspicious asymmetries, with and without an ultrasound correlate. This explains the significantly higher number $(p 0.001)$ of malignant lesions $(257 / 380,68.4 \%)$ than what was reported in previous studies [16-18].

The authors started the analysis of the LEMI by reporting the breast density with high-density breasts constituted the majority (72.6\%) of the cases. The lowenergy mammography images showed a positive and negative likelihood ratio of 1.54 and 0.37 , respectively, and an overall accuracy of $71 \%$. The poor results were mainly attributed to the predominant heterogeneous dense breast parenchyma among the study population which resulted in both over- and underestimation of the nature of BAs. The associated diffuse edema pattern that accompanied inflammatory breast lesions also increased the number of misinterpreted lesions resulting in 21/66 false-positive cases (31.8\%).

The role of incorporating ultrasound in the evaluation of breast asymmetries is not clearly defined in literature. Targeted ultrasound examination is usually performed to clarify the benign or malignant lesions underlying breast asymmetries. The presence of an underlying ultrasound correlate increases the level of suspicion for malignancy. However, in a study performed by Leung and Sickles [19], they affirmed that the lack of a ultrasound 

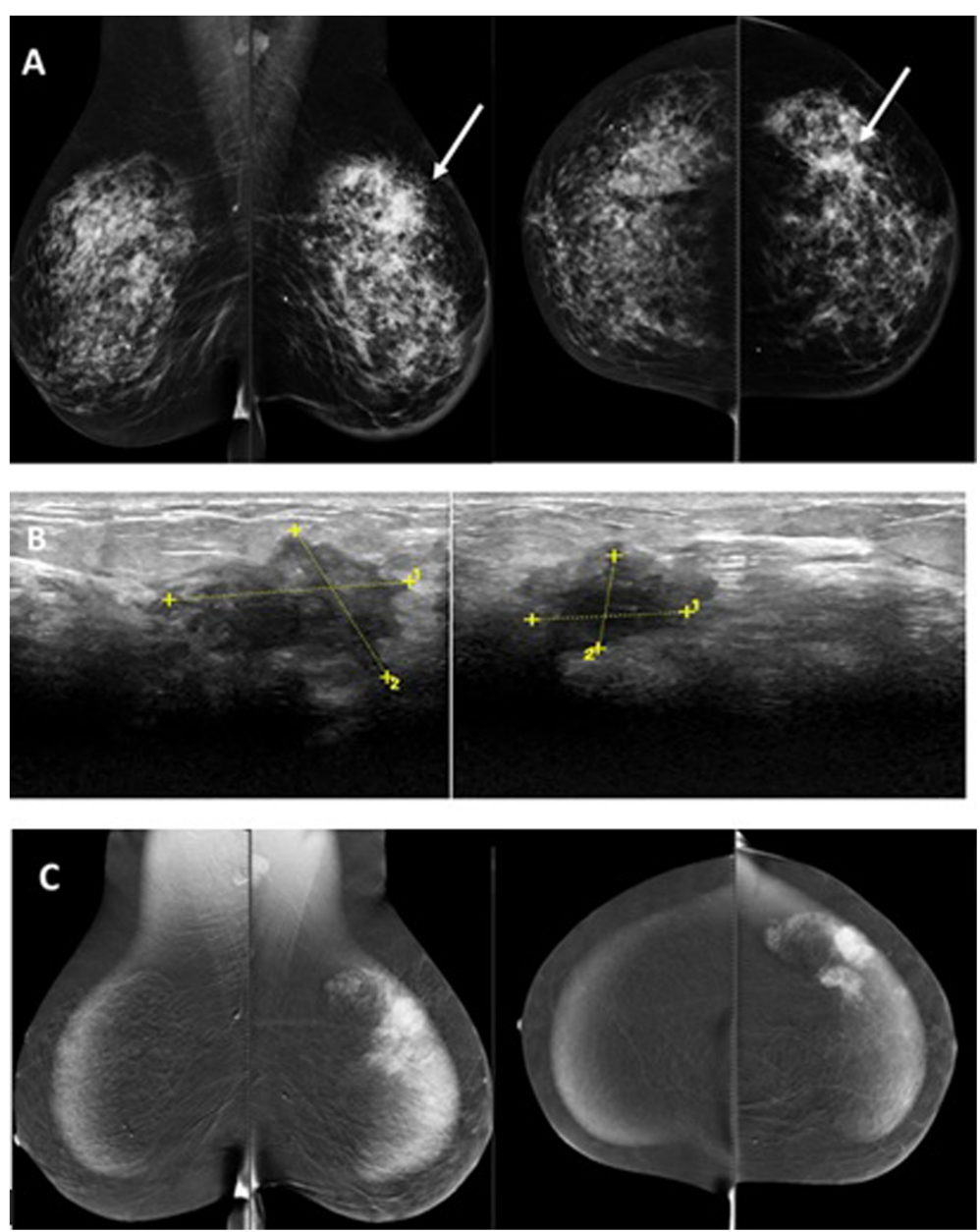

Fig. 3 Focal asymmetry associated with diffuse skin thickening (arrow) in a heterogeneous dense parenchyma seen in CC and MLO views (a). An evident underlying malignant ultrasound correlate was seen (b). CESM was performed to identify the actual extent of the malignant lesion (c). Wider tumor extension was identified on the contrast images. Biopsy revealed IDC2

(US) correlate which was reported in 5/21 malignant lesions in their study should not exclude biopsy or further imaging.

In the current study, the authors reported no ultrasound correlate in 90/380 (23.7\%) BAs, an indeterminate ultrasound correlate in 61/380 (16\%) BAs, and a malignant correlate in 229/380 (60.3\%) BAs. Adding ultrasound improved the overall accuracy of assessment to become $80.5 \%$ with the heterogeneous dense parenchyma and inflammatory breast lesions still contributing to a considerable number of FP and FN results.

Lee and coauthors $[10,12,13]$ found that MRI was a convenient modality for mammographic findings that were not adequately localized with mammography and US [20]. Areas showing enhancement may also guide for further biopsy intervention. CESM is an easier and cheaper alternative to mammography with comparable reported sensitivity and specificity.
In accordance to the study performed by Kamal and colleagues, the current study confirmed that nonenhancement strongly correlated with a benign pathology ( $p$ value 0.05) [21]. Another advantage of the contrast images over the mammography images is that the heterogeneous dense parenchyma did not correlate with a corresponding degree of BPE $(R 0.37$, $p$ value 0.00001$)$. Similar correlation was reported in several studies addressing parenchymal enhancement on MRI [22-24]. The absence of parenchymal enhancement on the contrast images resulted in a significant drop in the FP and FN results with an overall accuracy reaching $88.5 \%$. The calculated positive and negative likelihood ratio of the contrast images was 3.88 and 0.07 , respectively.

The subtracted breast parenchyma allowed for better lesion extension and multiplicity identification. A wider extension was identified in 42 asymmetries, and additional lesions were identified in another 12 asymmetries; 


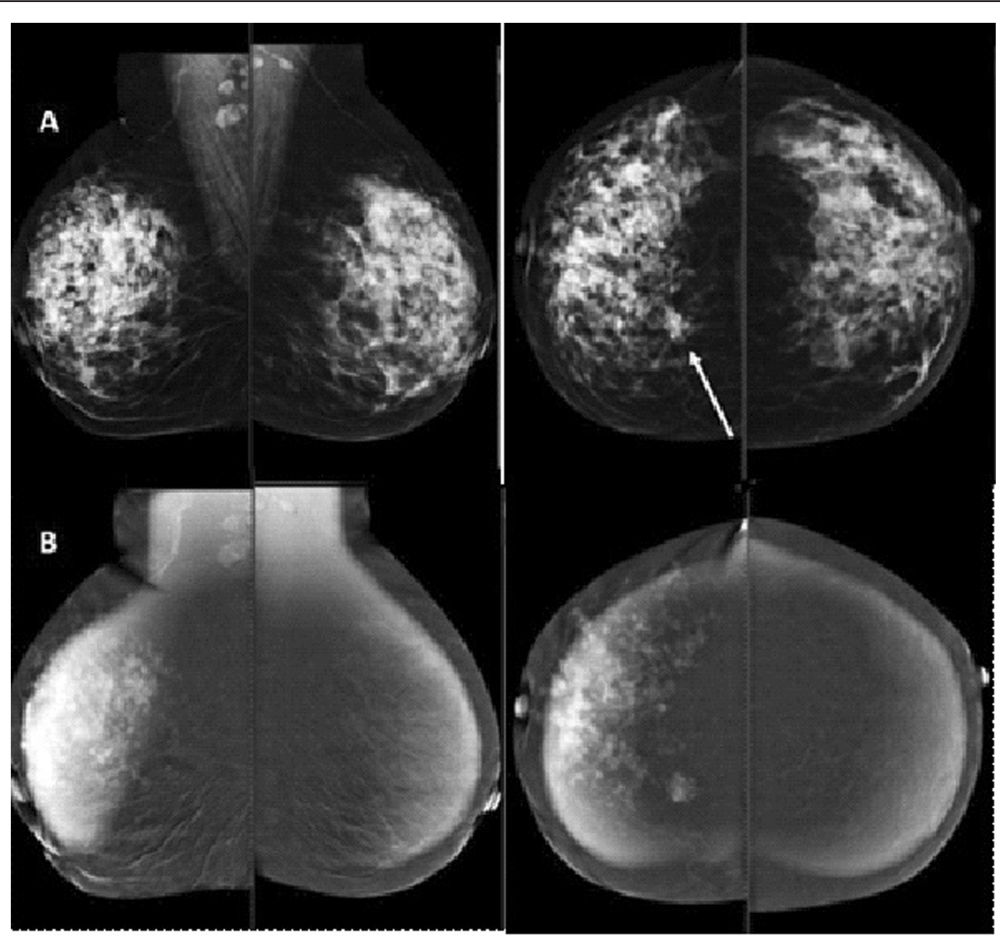

Fig. 4 Deeply seated lobular carcinoma presenting as a single view asymmetry (arrow) on the low-energy images in a heterogeneous dense breast parenchyma (a). A much wider tumor extension was identified on the contrast images (b). Patient management changed from conservative surgery to modified radical mastectomy

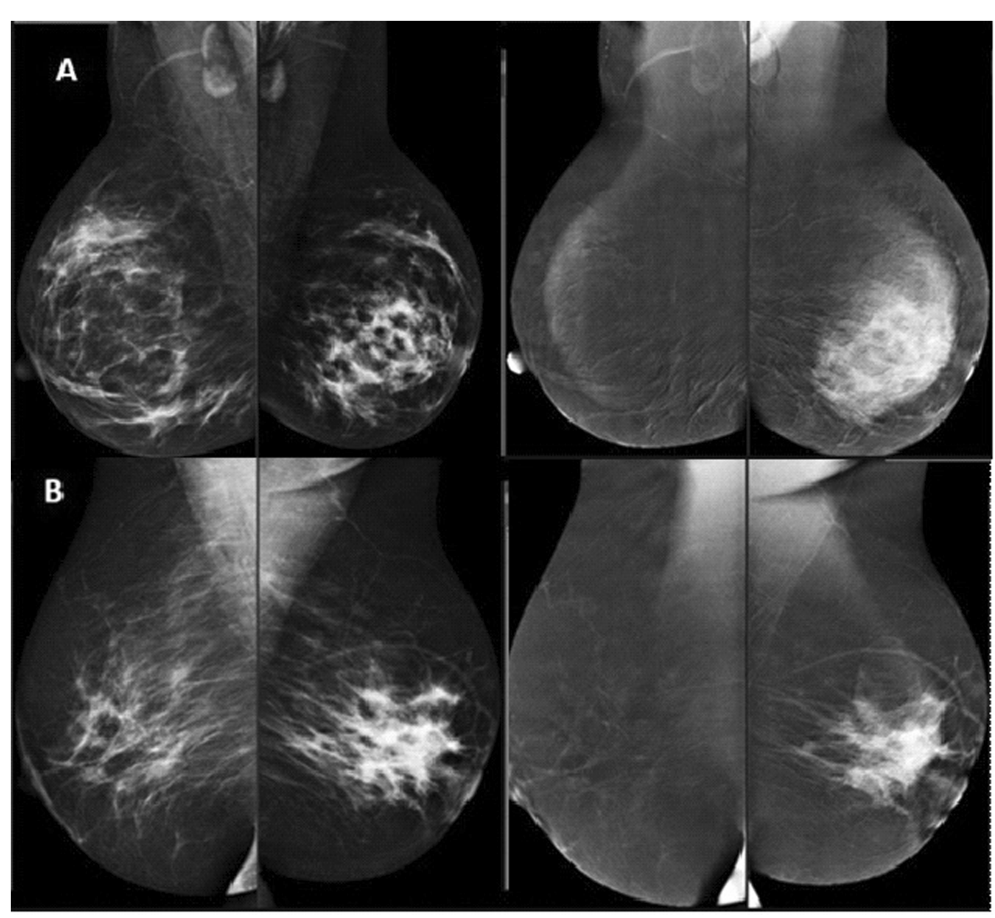

Fig. 5 Two different false-positive inflammatory lesions on both the low- and subtracted high-energy images. Biopsy revealed granulomatous mastitis (a) and periductal mastitis (b) 
this had a major influence on the accuracy of patient management.

Combining the sonomammography and contrast images had an impact on reducing the false-negative results, but no similar impact was perceived on the falsepositive results. This was mainly attributed to the intense enhancement that accompanied inflammatory breast lesions in a pattern that mimicked malignant lesions. The overall accuracy of the combined assessment reached $91.8 \%$.

\section{Conclusion}

Combining the ease of doing a mammogram and the merits of contrast, CESM should be considered in the diagnostic work-up of breast asymmetries not resolved on an initial combined mammography and targeted ultrasound study especially in the presence of a heterogeneous dense breast parenchyma. CESM helps in asymmetry characterization and can help in identifying the actual size, multiplicity, and extension of malignant lesions. However, the accuracy of CESM becomes questionable in the presence of inflammatory breast lesions due the overlap of enhancement patterns between inflammatory and malignant lesions.

\section{Abbreviations}

BAs: Breast asymmetries; BPE: Background parenchymal enhancement; CESM: Contrast-enhanced spectral mammogram; HEMI: High-energy mammography images; LEMI: Low-energy mammography images; US: ultrasound

\section{Acknowledgements}

No acknowledgements

\section{Authors' contributions}

RK wrote the manuscript. AM collected the patient data and is responsible for the correspondence to journal. SF contributed to the image processing and collection of patient images. EK participated in the design of the study and performed the statistical analysis. AR conceived of the study, participated in its design and coordination, and helped to draft the manuscript. AH was responsible for the revision of the draft from clinical point of view. MH contributed to the surgical data collection and statistical analysis. All authors have read and approved the manuscript.

\section{Funding}

No funding sources

\section{Availability of data and materials}

The datasets used and analyzed during the current study are available from the corresponding author on reasonable request.

\section{Ethics approval and consent to participate}

The study was approved by the ethical committee of a multidisciplinary "Breast Cancer Hospital," and informed written consent was taken from all subjects.

\section{Consent for publication}

All patients included in this research gave written informed consent to publish the data contained within this study.

\section{Competing interests}

The authors declare that they have no competing interests.

\section{Author details}

'Departemnt of diagnostic and interventional Radiology department, Faculty of medicine, Cairo University, Al Kasr Al Aini, Al Manial, Cairo Governorate, Egypt. ${ }^{2}$ Department of Diagnostic and Interventional Radiology, National Cancer Institute, Cairo University, 1 Kasr Elainy Street, Fom Elkalig, Cairo, Egypt. ${ }^{3}$ Department of Medical Oncology, National Cancer Institute, Cairo University, 1 Kasr Elainy Street, Fom Elkalig, Cairo, Egypt. ${ }^{4}$ Department of Surgical Oncology, National Cancer Institute, Cairo University, 1 Kasr Elainy Street, Fom Elkalig, Cairo, Egypt.

Received: 5 July 2019 Accepted: 30 August 2019

Published online: 07 October 2019

References

1. Friedman PD, Swaminathan SV, Herman K, Kalisher L (2006 Aug) Breast MRl: the importance of bilateral imaging. American Journal of Roentgenology 187(2):345-349

2. Youk JH, Kim EK, Ko KH, Kim MJ (2009 Jan) Asymmetric mammographic findings based on the fourth edition of BI-RADS: types, evaluation, and management. Radiographics 29(1):e33

3. Samardar P, de Paredes ES, Grimes MM, Wilson JD (2002 Jan) Focal asymmetric densities seen at mammography: US and pathologic correlation. Radiographics 22(1):19-33

4. Chesebro AL, Winkler NS, Birdwell RL, Giess CS (2016 Feb 19) Developing asymmetries at mammography: a multimodality approach to assessment and management. RadioGraphics 36(2):322-334

5. Sickles EA, d'Orsi CJ, Bassett LW, Appleton CM, Berg WA, Burnside ES. Acr birads ${ }^{\circledast}$ mammography. ACR BI-RADS ${ }^{\oplus}$ atlas, breast imaging reporting and data system. 2013;5.

6. Kopans DB, Swann CA, White G, McCarthy KA, Hall DA, Belmonte SJ, Gallagher W (1989 Jun) Asymmetric breast tissue. Radiology 171(3):639-643

7. Gilbert FJ, Tucker L, Young KC (2016 Feb 1) Digital breast tomosynthesis (DBT): a review of the evidence for use as a screening tool. Clinical radiology 71(2):141-150

8. Brenner RJ (2001) Asymmetric densities of the breast: strategies for imaging evaluation. Semin Roentgenol 36(3):201-216

9. Shetty MK, Watson AB (2002) Sonographic evaluation of focal asymmetric density of the breast. Ultrasound Q 18(2):115-121

10. Fallenberg EM, Dromain C, Diekmann F, Engelken F, Krohn M, Singh JM, Ingold-Heppner B, Winzer KJ, Bick U, Renz DM (2014) Contrast-enhanced spectral mammography versus MRI: initial results in the detection of breast cancer and assessment of tumour size. Eur Radiol 24:256-264

11. Morris EA, Comstock CE, Lee CH et al (2013) ACR BI-RADS ${ }^{\circledR}$ magnetic resonance imaging. In: ACR BI-RADS ${ }^{\star}$ atlas, breast imaging reporting and data system. American College of Radiology, Reston, VA

12. Kamal R (2016, issue) Breast imaging in Egypt and contrast-enhanced spectral mammography: an emerging promising breast imaging modality. SBI Newsletter (2):22-23

13. Dromain C, Balleyguier C, Muller S, Mathieu M, Rochard F, Opolon P, Sigal R (2006) Evaluation of tumor angiogenesis of breast carcinoma using contrastenhanced digital mammography. AJR Am J Roentgenol 187:W528-W537

14. Dromain C, Thibault F, Muller S, Rimareix F, Delaloge S, Tardivon A, Balleyguier C (2011) Dual-energy contrast-enhanced digital mammography: initial clinical results. Eur Radiol 21:565-574

15. Dromain C, Thibault F, Diekmann F, Fallenberg E, Jong RA, Hendrick RE, Tardivon A, Toledano A (2012) Dual-energy contrast-enhanced digital mammography: initial clinical results of a multireader, multicase study. Breast Cancer Res 14(3):R94

16. Sickles EA (1986) Mammographic features of 300 consecutive non palpable breast cancers. AJR Am J Roentgenol 146(4):661-663

17. Giess CS, Frost EP, Birdwell RL (2014) Interpreting one-view mammographic findings: minimizing callbacks while maximizing cancer detection. RadioGraphics 34(4):928-940

18. Sickles EA (1998) Findings at mammographic screening on only one standard projection: outcomes analysis. Radiology 208(2):471-475

19. Leung JW, Sickles EA (2007) Developing asymmetry identified on mammography: correlation with imaging outcome and pathologic findings. AJR Am J Roentgenol 188(3):667-675

20. Lee CH, Smith RC, Levine JA, Troiano RN, Tocino I (1999) Clinical usefulness of MR imaging of the breast in the evaluation of the problematic mammogram. AJR 173:1323-1329 
21. Kamal RM, Helal MH, Wessam R, Mansour SM, Godda I, Alieldin N (2015 Jun 1) Contrast-enhanced spectral mammography: impact of the qualitative morphology descriptors on the diagnosis of breast lesions. European journal of radiology. 84(6):1049-1055

22. King V, Brooks JD, Bernstein JL, Reiner AS, Pike MC, Morris EA (2011 Jul) Background parenchymal enhancement at breast MR imaging and breast cancer risk. Radiology. 260(1):50-60

23. Morris EA (2007 Sep 1) Diagnostic breast MR imaging: current status and future directions. Radiologic clinics of North America. 45(5):863-880

24. Cubuk R, Tasali N, Narin B, Keskiner F, Celik L, Guney S (2010 Apr 1) Correlation between breast density in mammography and background enhancement in MR mammography. La radiologia medica. 115(3):434-441

\section{Publisher's Note}

Springer Nature remains neutral with regard to jurisdictional claims in published maps and institutional affiliations.

\section{Submit your manuscript to a SpringerOpen ${ }^{\circ}$ journal and benefit from:}

- Convenient online submission

- Rigorous peer review

- Open access: articles freely available online

High visibility within the field

- Retaining the copyright to your article

Submit your next manuscript at $\boldsymbol{\wedge}$ springeropen.com 